\title{
Evaluation of Accurate Indoor Localization Systems in Industrial Environments
}

\author{
Abdulkadir Karaagac, Jetmir Haxhibeqiri, Matteo Ridolfi, Wout Joseph, Ingrid Moerman, Jeroen Hoebeke \\ Ghent University - imec, IDLab, Department of Information Technology \\ Technologiepark Zwijnaarde 15, B-9052 Ghent, Belgium \\ \{abdulkadir.karaagac, jetmir.haxhibeqiri, matteo.ridolfi, wout.joseph, ingrid.moerman, jeroen.hoebeke\}@ugent.be
}

\begin{abstract}
Due to the fast emergence of location-based services and the absence of a widely adopted localization technology for indoor environments, Indoor Localization Systems have become a central topic of research in the last decade. Although there is a significant amount of research targeting indoor localization technologies and their performance, most of these efforts only focus on theory, system design or evaluation in non-industrial environments, usually offices or healthcare spaces. In this work, a detailed performance evaluation of two commercially available accurate localization technologies, based on Bluetooth Low Energy (LE) and Ultra-wideband (UWB), in an industrial environment is presented to create an experimental understanding of their behaviour in similar conditions and to investigate their potential to be used in industrial applications with concrete localization requirements. For this purpose, these localization technologies are examined with respect to various performance criteria in several scenarios in a real industrial site.
\end{abstract}

Keywords - Indoor Localization, Industrial Environment, Performance Evaluation, UWB, Bluetooth LE

\section{INTRODUCTION}

In recent years, the demand for indoor localization solutions has been rapidly expanding in many fields [1-3]. Particularly, there is a significant potential for such applications in the industrial world to improve automation and increase efficiency, including tracking goods and robots, operator guidance, etc. However, industrial environments impose additional challenges for localization systems because of the large number of active and passive perturbations, especially shadowing and multipath effects due to metallic objects $[1,4,5]$. Therefore, there is a need for further evaluation and validation of current localization technologies in real-life industrial conditions.

In order to meet strict requirements of industrial applications, even in harsh environments, two promising commercially available localization technologies have been selected for evaluation in an industrial environment; namely Royal FloraHolland Flower Auction Center in the Netherlands. The first system is based on ultra-wideband (UWB) technology, which is well known for its capability to provide highly accurate positioning even in the presence of severe multipath. Moreover, since UWB is operating in a totally different frequency band, it does not interfere with existing widely used RF technologies [6]. These features make UWB a suitable choice for critical positioning applications that require highly accurate results. The second technology of choice is an Angle-of-Arrival (AoA) based Bluetooth Low Energy (BLE) localization technology which can provide real-time highly accurate positioning information. Although it is mainly based on Bluetooth LE, its proprietary design and the use of special antennas facilitate the realization of an accurate and robust indoor localization system.
In scientific literature, a large number of research is available regarding Indoor Localization Systems and their performance. However, most of these efforts only focus on theory, system design, survey of localization techniques or evaluation in non-industrial environments, usually offices or healthcare spaces [7-10]. The main contributions of this paper are (i) a detailed performance evaluation and comparison of two different promising accurate localization technologies, Bluetooth LE and UWB, in an industrial environment, (ii) an experimental understanding of their behavior in similar conditions and (iii) investigation of their potential to be used in industrial applications with given localization requirements

The remainder of this paper is organized as follows. Section II briefly describes related work. In Section III, the evaluation environment is described in detail. Section IV presents the technology overview, system setups, measurement scenarios and results for selected localization technologies. Afterwards, Section V discusses the performance of both technologies and their adoption potential for specific localization applications. Finally, Section VI concludes the paper.

\section{RELATED WORK}

Several studies have been conducted that investigate the challenges and solutions for localization technologies in industrial-like environments $[1,7,11,12]$. However, unlike the focus of this paper, they generally study fingerprinting based technologies or solutions, which realize lower level of accuracy, or lack a comparison in real-life conditions.

In [1], Holzl et al. evaluate the impact of electromagnetic influences and shadowing effects in an industrial environment on the accuracy of positioning systems that are based on Wi-Fi fingerprinting and dead reckoning. Similarly, in [7], a Wi-Fi fingerprinting method is studied for an indoor positioning application in a real construction site and a practical labor tracking application is developed. On the other side, [11] investigates the effect of environmental conditions on the performance of three selected localization solutions (fingerprinting, time-of-arrival and RSSI based) by using the same evaluation methodology in three different environments including two office-like areas and a testbed area with metal walls and obstacles. [12] studies the fundamental limits of UWB ranging accuracy via a survey of time-base ranging UWB signals in multipath environments. Also, the main sources of error in Time-of-Arrival (TOA) estimation, such as multipath, interference and clocks drift, are discussed. A study on an UWB indoor localization system for applications in industrial buildings is presented in [13]. Also, [14] presents an evaluation of an indoor localization solution based on TOA in a challenging largescale wireless testbed which consists of metallic walls and several metal obstacles such as vertical and horizontal pipes. 


\section{III.MEASUREMENT ENVIRONMENT}

The Royal FloraHolland Auction Center [15] is located in Naaldwijk, in the Netherlands. In general, the Auction Center, spanning a total area of more than $300.000 \mathrm{~m}^{2}$, consists of various sections including auction halls, storing, buffering and distribution zones with different characteristics. Figure 1 shows one of the buffering zones where the flower trolleys are automatically buffered and then distributed manually by operators. Due to its dynamic nature and also for practical convenience, this area was selected as main testing area where we operated our measurements.

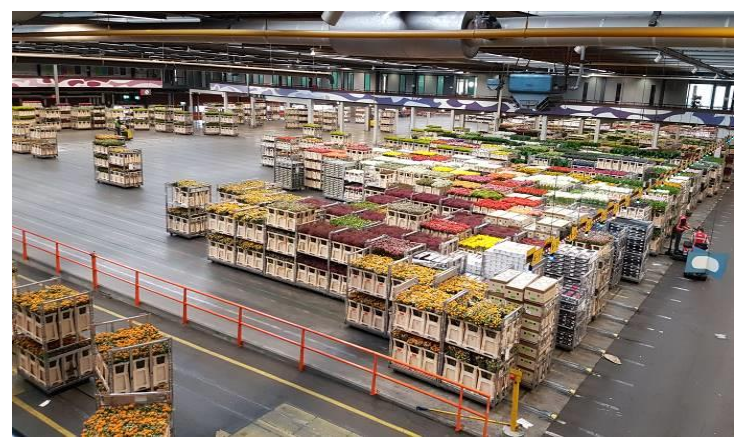

Fig. 1: Measurement Environment - FloraHolland Trollley Buffering Area

The floor plan is presented in Figure 2, comprising the measurement area, which is a $60 \times 46 \mathrm{~m}$ non-flat area with a maximum floor height difference of $0.7 \mathrm{~m}$. The ceiling height is varying between $8 \mathrm{~m}$ to $10 \mathrm{~m}$, so it can be counted as a halfopen area. On the sides of the measurement area, there are sideways (at a height of $5.6 \mathrm{~m}$ ) which are used for the installation of both localization systems in order to ease the installation and calibration process. The measurement area has a very dynamic and mobile nature due to auction trolleys, full of plants and flowers, which are moved and buffered within the buffering zone. These aluminum trolleys are the logistic tools to transport goods within the Royal FloraHolland [15]. The target use case for this setting is the tracking and monitoring of transporting trolleys within the whole industrial area with trolley-level accuracy, i.e. $0.5 \mathrm{~m}$.

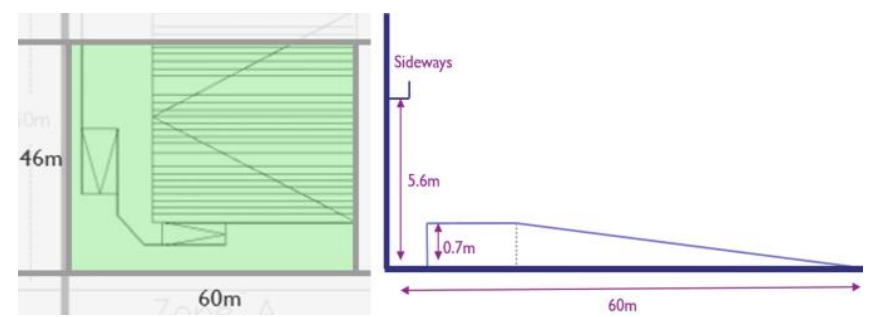

Fig. 2: Floor Plan of the Measurement Area

\section{Measurement Setup ANd Results}

Since the selected systems rely on different techniques and wireless technologies, we applied two different measurement setups and performed an extensive evaluation of their performance in various scenarios. Firstly, a detailed description is provided for each technology. Then, the system setup and scenarios are explained, followed by the results in terms of several performance metrics including accuracy, availability and scalability.

\section{A. UWB Localization}

Ultra-wideband (UWB) is a promising technology in the field of indoor positioning and is well known for its capability to provide very precise positioning which makes it a suitable choice for critical applications that require highly accurate position information [6].
The selected UWB technology is a commercially available localization system which can be used in both indoor and outdoor applications. For outdoor environments, UWB signals have a maximum range of $100 \mathrm{~m}$ in clear lineof-sight (LOS). In an indoor industrial environment, it is expected that the localization performance decays due to interferences, shadowing and multipath propagation [14]. However, since the system operates between $3.5-6.5 \mathrm{GHz}$, it does not interfere with other RF systems such as Wi-Fi and Bluetooth. The localization system supports position update rates between $5-50 \mathrm{~Hz}$ for a single tag depending on the UWB configuration such preamble length and transmission rate. For multiple tags, the maximum update rate is divided by the number of tags.

Like many UWB positioning technologies, the considered localization system is based on the utilization of time-of-flight of the RF signals to calculate the distance between the target, 'tag', and reference devices, 'anchors'. First of all, the anchor boards are placed at well-known fixed positions in the deployment area and this position information is hardcoded in 'tag' device. Next, the tag starts measuring its distance to anchor nodes via a 2-way ranging mechanism. After all, these ranging measurements are used by the tag to compute the estimated position by using a multi-lateration algorithm. At the time of the measurements, the system was only supporting $2 \mathrm{D}$ and $2.5 \mathrm{D}$ positioning where the height of the tag needs to be fixed and known by the system. This is a limiting feature for our setup in a nonflat area with up to $0.7 \mathrm{~m}$ ground level variation.

\section{1) System Setup}

For the evaluation of the UWB localization system, we used 4 anchors, fixed around the measurement area, and 1 mobile tag which was localized as it was moved around. The localization data was collected by the mobile tag and retrieved at the end of each measurement.

Due to practical reasons, the anchors were fixed on the sidebars of the sideways at a height of $5.6 \mathrm{~m}$ from the ground. Before deploying the anchors, we measured the maximum operational system range to determine the best anchor positions and the area that the system could cover. As it is also presented in scenario IV.A.1.i, the system was able to detect the distance at most $30 \mathrm{~m}$ in a stable and reliable manner. By considering system range and other practical conditions, we designed the system setup and deployed the anchors as presented in Figure 3. In the figure, the coverage of each anchor is presented by a circle around it. Figure 3 shows that the localization system had a good coverage over almost half of the buffering zone. This area has a very dynamic and harsh nature for our measurements due to the trolleys, which were transported to the buffering zone.

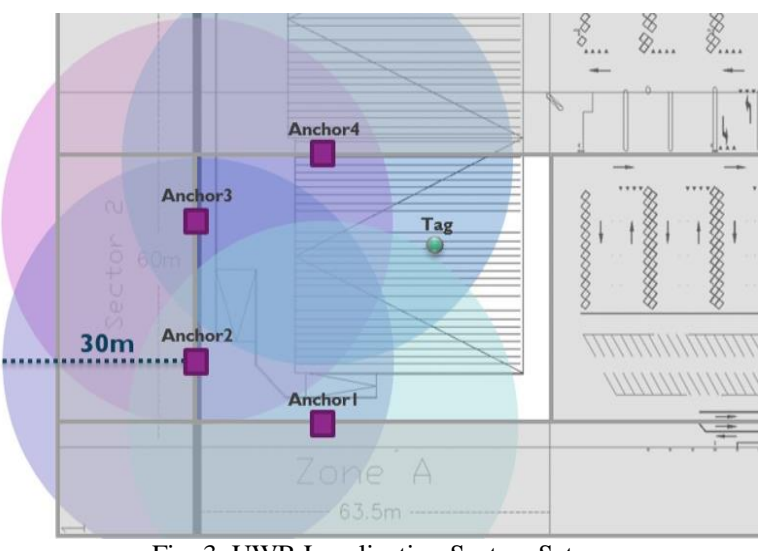

Fig. 3: UWB Localization System Setup 


\section{2) Measurement Scenarios and Results}

In order to obtain a better understanding of the localization system under test, we performed measurements in 4 scenarios with different conditions and performance metrics. An overview of these scenarios is given in Figure 4.

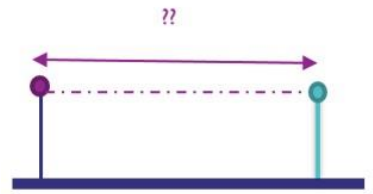

(a)

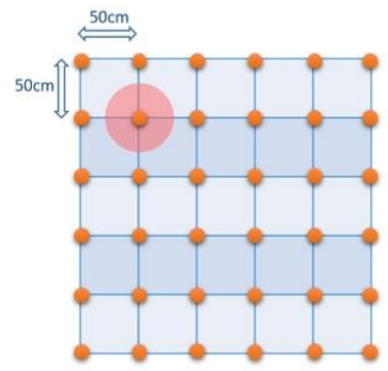

(c)

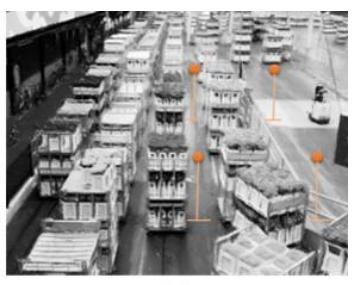

(b)

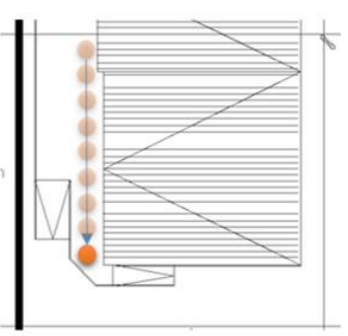

(d)
Fig. 4: Measurement Scenarios for UWB-based Localization System

(a) Ranging accuracy (b) Ranging accuracy in dynamic environment (c) Postioning accuracy (d) Positioning accuracy in mobility

\section{i) Ranging Accuracy in Static Environment (figure 4-a)}

The aim of this test was to investigate the operational range of the localization system and its coverage capabilities for positioning applications. For this purpose, the ranging measurements of the tag to one anchor were collected for different distances, with assured Line-of-Sight (LOS), until the maximum achievable operational range. For these measurements, the $3244.8-3744 \mathrm{MHz}$ frequency band was used for ranging signals with a preamble length of 4096 and bit rate of $110 \mathrm{kbps}$.

The range information was collected for distances between $10 \mathrm{~m}$ and $35 \mathrm{~m}$ and the distribution of the ranging error, in $\mathrm{mm}$, compared to the real distance is provided in Figure 5. Also, the average absolute error and standard deviation values for ranging measurements are provided in Table 1. These results show that, until $35 \mathrm{~m}$, the average ranging error is less than $50 \mathrm{~mm}$ with standard deviation around $50 \mathrm{~mm}$. A drastic performance drop is observed after $30 \mathrm{~m}$ which resulted in an average absolute error value of $226 \mathrm{~mm}$ at $35 \mathrm{~m}$. Also, the system availability deteriorated to $43.4 \%$ which means the tag was not able to detect the range more than half of the times. At $40 \mathrm{~m}$ and further distances, the tag was completely out of the anchor range and it was not able to provide any ranging information.
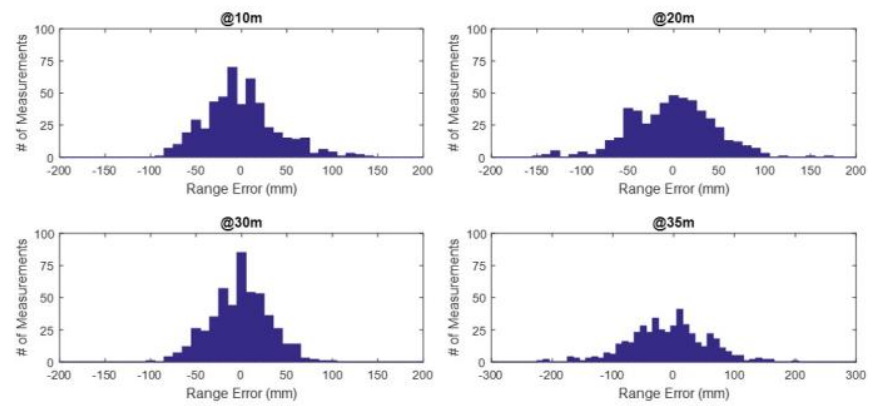

Fig. 5: Distribution of UWB Ranging Errors for different distances
TABLE I.

UWB - RANGING ERROR AND SYSTEM AVAILABILITY

\begin{tabular}{cccc}
\hline Distance & $\begin{array}{c}\text { Avg Abs Err } \\
(\mathbf{m m})\end{array}$ & $\begin{array}{c}\text { St Dev Err } \\
(\mathbf{m m})\end{array}$ & $\begin{array}{c}\text { Availability } \\
(\mathbf{\%})\end{array}$ \\
\hline $\mathbf{1 0}$ & 32.3 & 44 & 97 \\
$\mathbf{1 5}$ & 50.6 & 73 & 96,1 \\
$\mathbf{2 0}$ & 38.0 & 54 & 95,23 \\
$\mathbf{2 5}$ & 36.3 & 62 & 96,1 \\
$\mathbf{3 0}$ & 25.8 & 42 & 92,59 \\
$\mathbf{3 5}$ & 226.0 & 698 & 43,4 \\
\hline
\end{tabular}

These measurements show that the system can only detect the distances, in a stable and reliable manner, to the anchors which are in $30 \mathrm{~m}$ range. Since the coverage determines the number of anchor devices required for larger applications, these results also give an idea about the system installation cost for the localization technology.

\section{ii) Ranging in Dynamic Environment (figure 4-b)}

The objective of this scenario was to evaluate the accuracy, reliability and scalability of the localization system under test in a more dynamic and harsh environment. This test was executed in the environment with a very dynamic nature due to auction trolleys, full of plants and flowers, which were distributed by several operators. The tag was positioned at three different points (all $18 \mathrm{~m}$ away from the anchor) among the trolley system track where the trolleys and operators were mostly in movement. LOS between the tag and anchor was assured in all three cases.

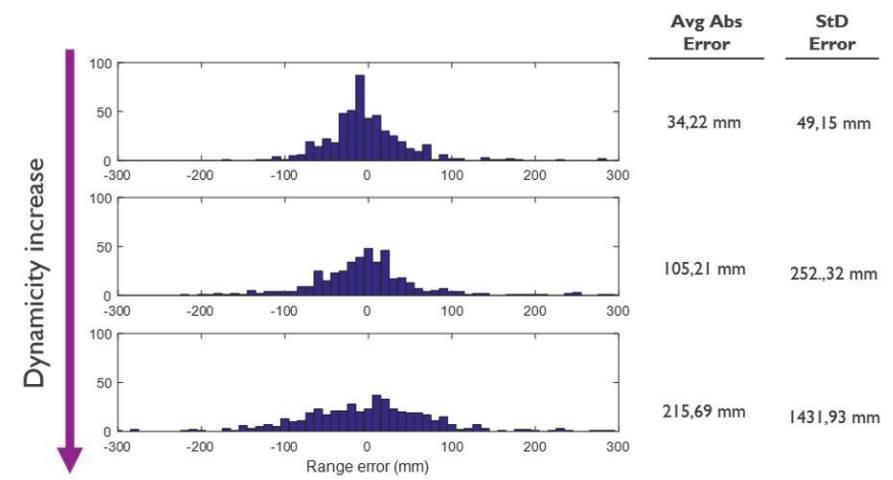

Fig. 6: UWB ranging accuracy with increased dynamicity

In addition to the distribution of the ranging error in millimeter, the average and standard deviation of the absolute ranging error for all three measurement points are provided in Figure 6. These measurements show that, as the amount of dynamicity increased, the ranging accuracy diminishes. Between the first and third measurement point, the average ranging error increased from $34.22 \mathrm{~mm}$ to $215.69 \mathrm{~mm}$. Such ranging errors might result in a more excessive deviation in positioning results. However, the localization system was still able to operate even in the most dynamic case with a relatively lower accuracy performance.

\section{iii) Positioning Accuracy (figure 4-c)}

The aim of this test was to investigate the positioning accuracy of the localization system. To do so, a mobile tag was located at 36 different positions which were $50 \mathrm{~cm}$ apart from each other. The results represent the position calculations solely based on the latest range measurements, in other words it does not use the current state for the next position calculation. Therefore, the effect of noises in measurements are not filtered and the outliers are still included. This test was executed when the environment had a static nature (no trolleys present) with minimum interference (no wireless activity due to operators). The outcome of this scenario gives an idea about the best achievable accuracy for this technology in such an industrial environment. 


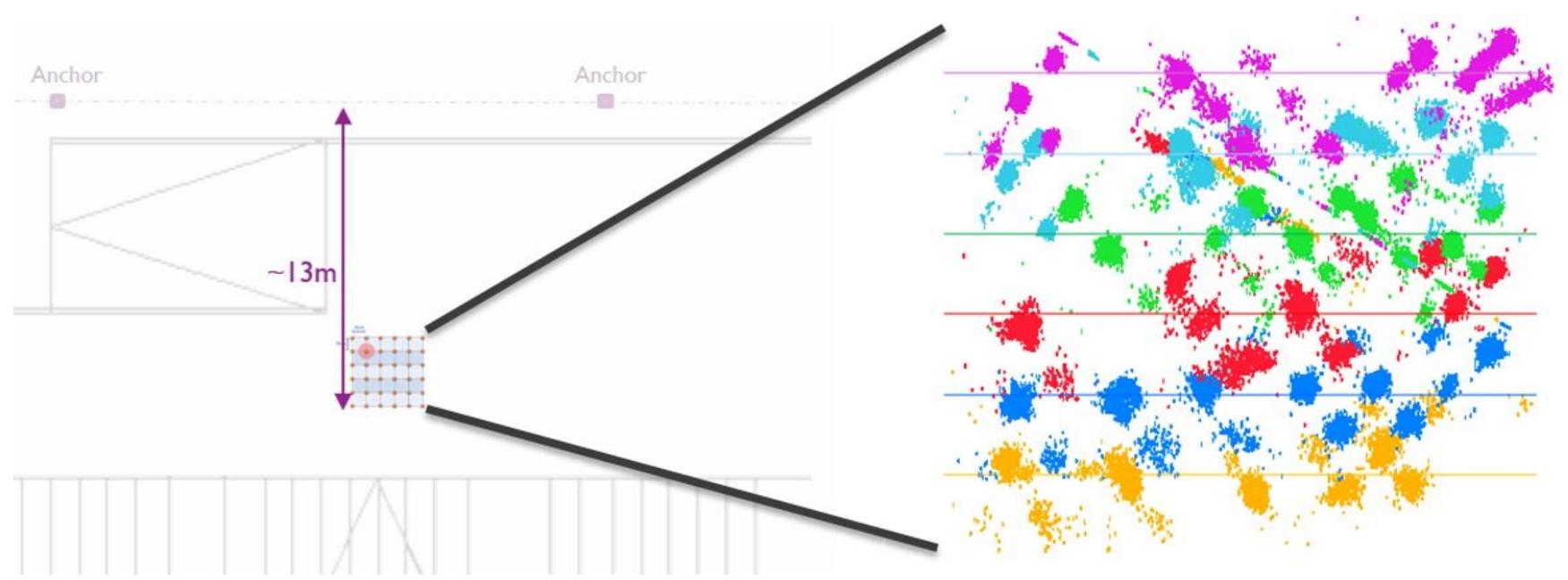

Fig. 7: The exact and measured points for UWB Position Accuracy Measurements

The measurement points and detected locations are presented in Figure 7. The measurements points which are aligned on the same line are illustrated with the same color. The position measurements in Figure 7 are not strictly precise and there are errors in measured positions. Sometimes it is not possible to differentiate the exact points that the tag was positioned at a certain time. These errors might be due to noise in the measurements which are not filtered by an advanced algorithm. In case of using more complicated and advance positioning algorithms with filters, a better and smoother positioning accuracy can be achieved. However, according to the results, sub-meter accuracy was acquired for $91 \%$ of the measurements which might be even sufficient for many applications.

\section{iv) Positioning with Mobility (figure 4.d)}

To understand how localization system performs in case of mobility, we set up a scenario where a tag was moved along a line with a constant speed.

The measured positions are provided in Figure 8. These results show that, in addition to accurate measurements (red), there are also some erroneous measurements (blue), which are not relevant to the movement track that tag followed. The temporary errors can be easily filtered via state estimators, e.g. Kalman filtering [16], and tracking algorithms which were not available in this localization system. A strange behavior in the measurements was that the erroneous measurements were aligned on a line towards anchors. We suspect that this happens when the node is not able to update the distance information for some anchors.

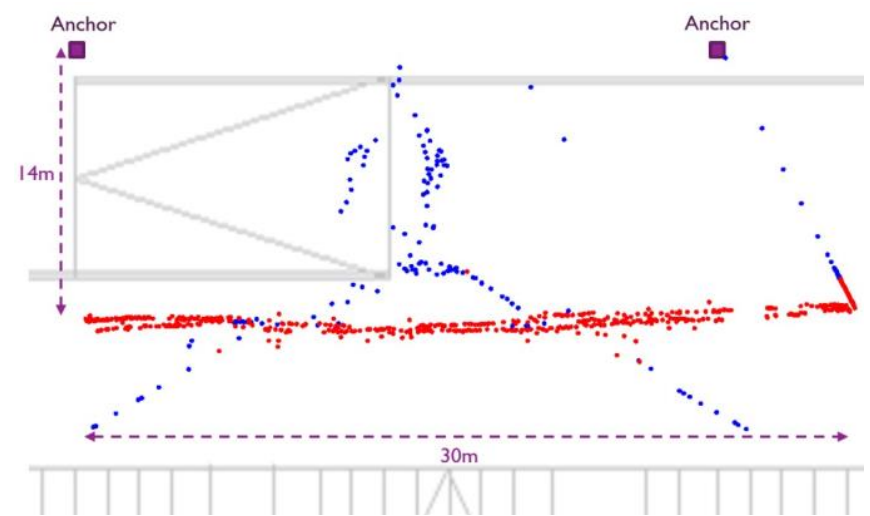

Fig. 8: UWB positioning accuracy in mobility

\section{B. Bluetooth Low Energy Localization}

A BLE based localization technology is the second system that we investigate in this work. Although it is mainly based on BLE technology, it also has a proprietary design which enables a more robust and reliable localization system compared to standard BLE. The system is able to use proprietary channels $(2401 \mathrm{MHz}$ and $2481 \mathrm{MHz})$ at the band edges of global $2.4 \mathrm{GHz}$ ISM communication band in order to avoid inter-system interferences with standard $\mathrm{Wi}-\mathrm{Fi}$ and Bluetooth. However, such a mode of operation introduces incompatibility with standard mobile devices.

This BLE-based localization system uses devices with special antennas, the 'anchors', to measure the Angle of Arrival (AOA) of a radio signal transmitted by a BLE 'tag'. The measurements are sent to a centralized positioning engine which uses algorithms to compute the position of the tag. The measurement height (assumption for tag height) is fixed and configured at system level. With one anchor, the system can perform 2D positioning of a tag (height is known) and two anchor devices are sufficient to do 3D positioning. The Positioning Engine only provides position information and a limited set of details about the measurements. Since we cannot retrieve any raw data (e.g. Angle of Arrival), we can only evaluate this system based on the post-processed position information provided.

According to the system description, the localization system can be configured with an update rate up to $100 \mathrm{~Hz}$, and latency down to $100 \mathrm{~ms}$. For BLE channels, the rate is limited to $10 \mathrm{~Hz}$ due to BLE regulations. Location update rate and latency are also dependent on the software license type provide by the system supplier.

\section{1) System Setup}

Due to coverage limitations and distinct system characteristics, we performed a different scheme for BLE localization system deployment compared to UWB. The sideway on the first floor was used in order to deploy the anchors, like for UWB. This choice also permitted us to reposition and re-orientate the anchors to calibrate the individual anchors and also overall system setup. Since the sideways are $5.6 \mathrm{~m}$ high and the trolleys are $2 \mathrm{~m}$ high, it can be expected to have at least $3.6 \mathrm{~m}$ height difference between the anchors and the tags mounted on the trolleys as presented in Figure 9. This height difference is crucial for the coverage and accuracy of the AoA-based localization technologies. 


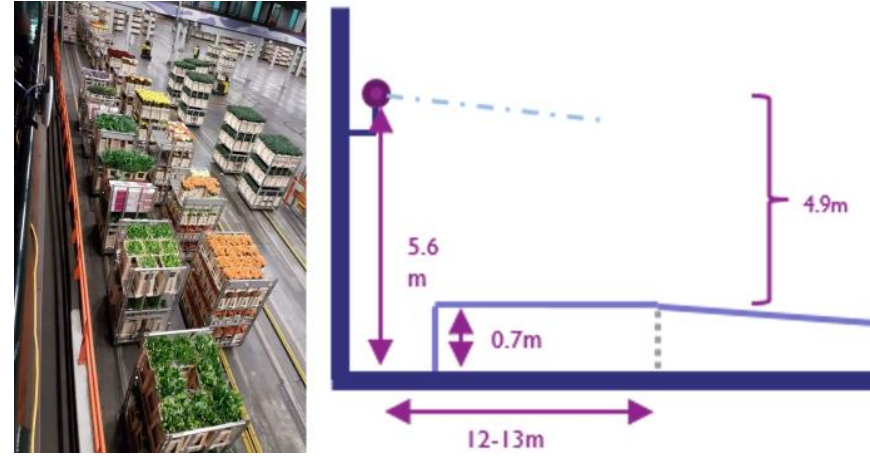

Fig. 9: Plan of the Measurement Area for BLE Localization System

For system installation, we used 5 anchor devices which can only cover a smaller sub-area within the whole buffering zone. The system planning and deployment tool was used to estimate the coverage and to optimize the position of the anchors accordingly. By taking practical (cabling) and physical conditions of the measurement area and the measurement scenarios in the consideration, we obtained the System Setup presented with a heat map in Figure 10.

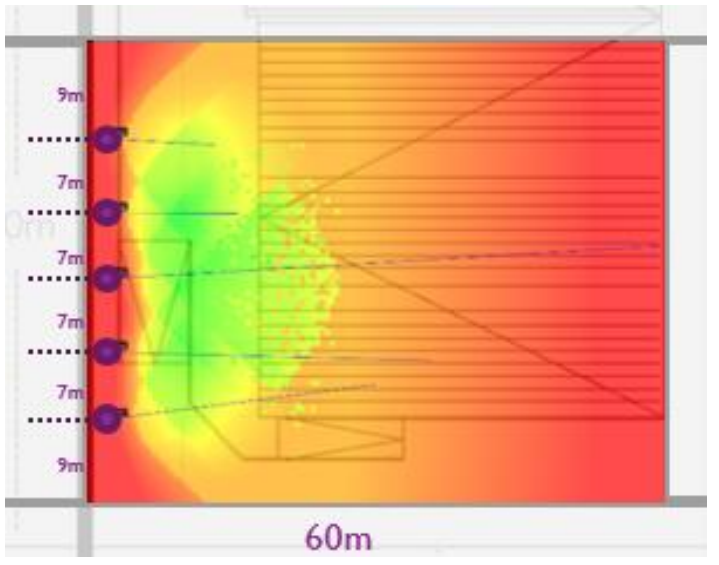

Fig. 10: Heatmap of BLE Localization System Coverage

\section{2) Measurement Scenarios and Results}

To have a better understanding about the performance of the AoA based BLE localization system, we had measurements in 6 different scenarios with varying conditions and performance metrics. The overview of these scenarios is shown in Figure 11. The detailed description of all these scenarios is given in the following sections.

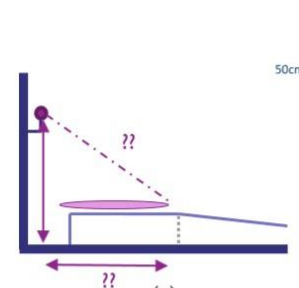

(a)

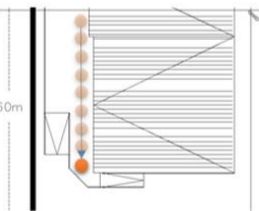

(d)

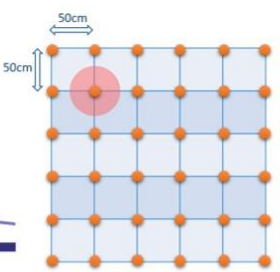

(b)

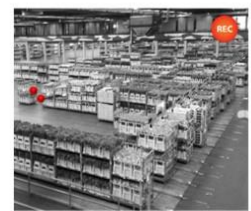

(e)

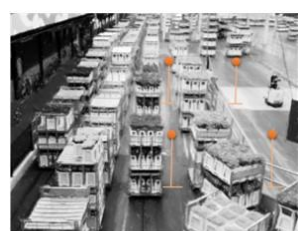

(c)

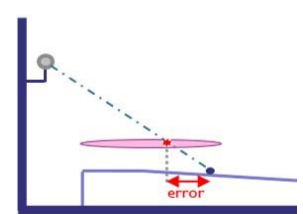

(f)
Fig. 11: Measurement Scenarios for BLE Localization System (a) System Coverage (b) Positioning Accuracy (c) Positioning in Dynamic Environment (d) Positioning Accuracy in Mobility (e) Multiple Tags

(f) The effect of height error

\section{i) Coverage Test (figure 11-a)}

The objective of this measurement was to understand the coverage of the BLE localization system with the available infrastructure. The outcome of this test was also beneficial for the evaluation of the technology's scalability and the estimation of its installation cost. In addition, the results of this test were also taken into account during the design of the measurements for other scenarios.

To be able to collect the coverage data, a tag was positioned at certain distances from the line where the anchors were aligned and the positioning information was collected at a centralized positioning engine. As presented in Figure 12, the system was able to locate the tag until $11 \mathrm{~m}$ from the line where the anchors were aligned. That means a separation of $13 \mathrm{~m}$ from the closest anchor and $15 \mathrm{~m}$ to two other anchors. For further points, the system failed to provide any positioning data. Therefore, we can conclude that this BLE localization system requires at most $15 \mathrm{~m}$ separation from at least two anchors for proper functioning. In addition, based on these observations, the following measurement scenarios were executed in the rectangular area with at most $11 \mathrm{~m}$ distance from the anchors' line.

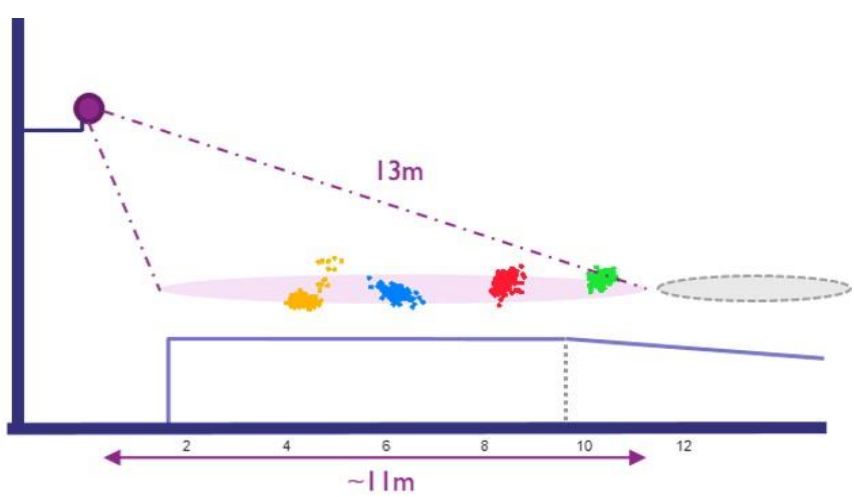

Fig. 12: Coverage of BLE Localization System

ii) Accuracy in Industrial Environment (figure 11-b)

In order to investigate the accuracy level of the localization systems in an industrial environment, as it is also presented in Figure 13, a mobile tag was located at 36 different positions which were $50 \mathrm{~cm}$ apart from each other and the furthest point was $7 \mathrm{~m}$ away from the line of anchors. Since this test took place when the environment had a static nature (no trolleys were present) and minimal interference (no wireless activity due to operators), the outcome of this test gives an idea about the best achievable accuracy for AoA based BLE localization technology in such industrial environments. The collected positioning information for every measurement point is demonstrated in Figure 13. For all points, the system was able to achieve a very accurate positioning performance $(\sim 20 \mathrm{~cm})$.

However, these results are an outcome of a tracking mechanism while the tags are static with guaranteed LOS and minimized environmental effects. That explains the very low variation and the absence of any outlier in the positioning data. In addition, the measurement area was in the range of 3 Anchor nodes. As such, this test has to be taken as the best performance of the localization system that can be achieved in such environments. 


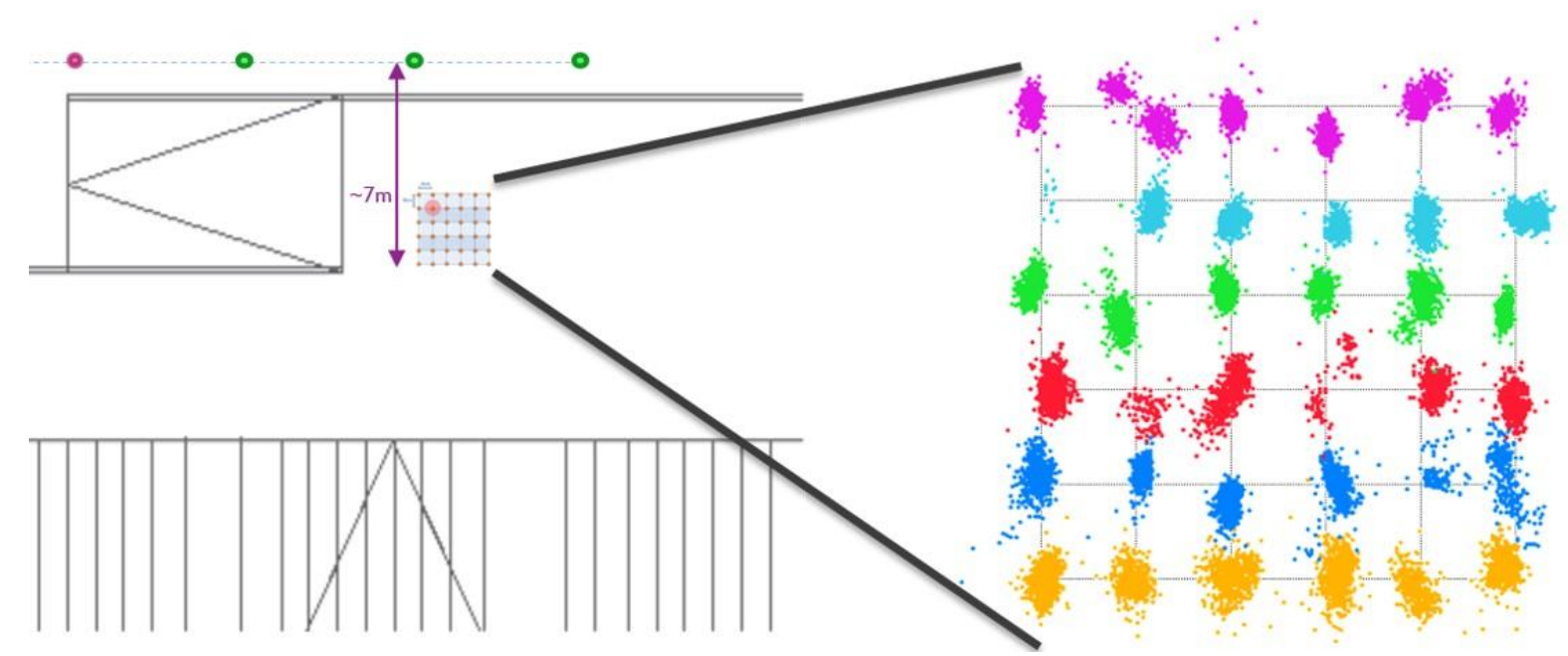

Fig. 13: The exact and measured points for BLE Position Accuracy Measurements

Another important observation is the issues we encountered with respect to the availability for certain points in the measurement area. Although we executed the same scenario for certain points multiple times, the system was not able to locate the tag for that position. However, after a little movement in any direction, it was able to locate it again. We think that this behavior is due to the very specific nature of that position, for instance destructive interference due to reflected waves might prevent the system to detect correct AoA to calculate the position of the tag.

iii) Performance in Dynamic Environment (figure 11-c)

The objective of this scenario was to evaluate the accuracy, reliability and scalability of the localization systems in a very dynamic and harsh environment. Therefore, this test was executed in a time period when many trolleys, full of flowers, were passing the measurement area and transferred to buffering zone. The tags were positioned on a fixed trolley surrounded by other mobile trolleys which are passing by. Firstly, the measurements were performed when the tags were located on top of the trolley which created a LOS between the anchors and the tag. Then, the tags were located on the side of the trolley which led to a NLOS scenario for our measurements. (a)

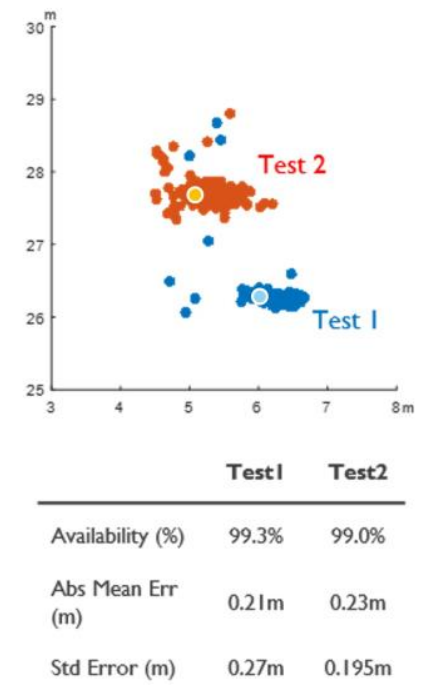

(b)

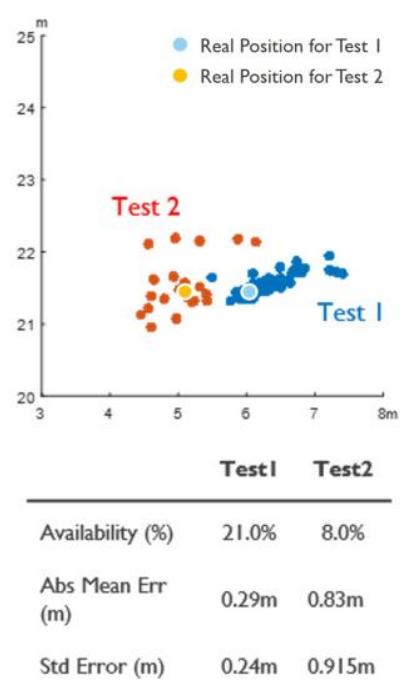

Fig. 14: BLE Localization Accuracy in Dynamic Environment (a) Line-Of-Sight \& On Top of the Trolleys (b) Non-Line-Of-Sight \& Sides of the Trolleys
Figure 14 shows the positioning measurements and system performance in terms of positioning accuracy and availability in case of $\operatorname{LOS}$ (a) and NLOS (b) between anchors and tags. These results show that the system was still able to perform very well in case of LOS. However, when there was no LOS, the system availability dropped drastically down to $8 \%$, while still having a good accuracy in case of availability.

\section{iv) Mobile Tag with Constant Speed (figure 11-d)}

To understand how the system performs in case of mobility, we set up a scenario where three tags are fixed on top of a trolley which is moved on a line, $5 \mathrm{~m}$ away from the anchors, with a constant speed. The measured positions with availability performance are provided in Figure 15. These results show that the system was able to provide accurate position updates, with $75 \%$ availability performance, even in case of mobility.

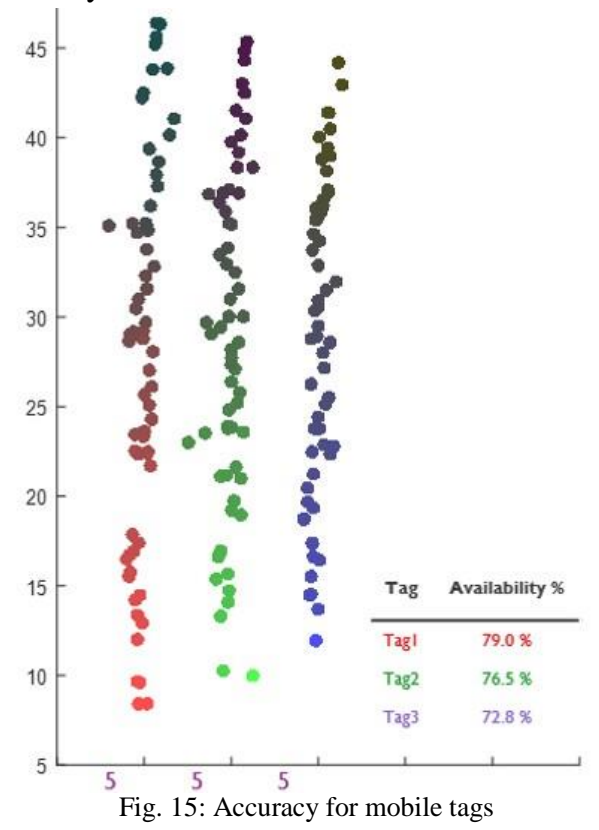

v) Multiple Tags - Visualization (figure 11-e)

To obtain a better visualization of the overall system accuracy, we executed a measurement scenario where three mobile tags were fixed on the same trolley and moved on along a $180 \mathrm{~m}$ track around the testing area with a speed of about $0.6 \mathrm{~m} / \mathrm{s}$. Meanwhile a camera recorded everything. The measurement area, movement track and the orientation of the tags are presented in Figure 16. 

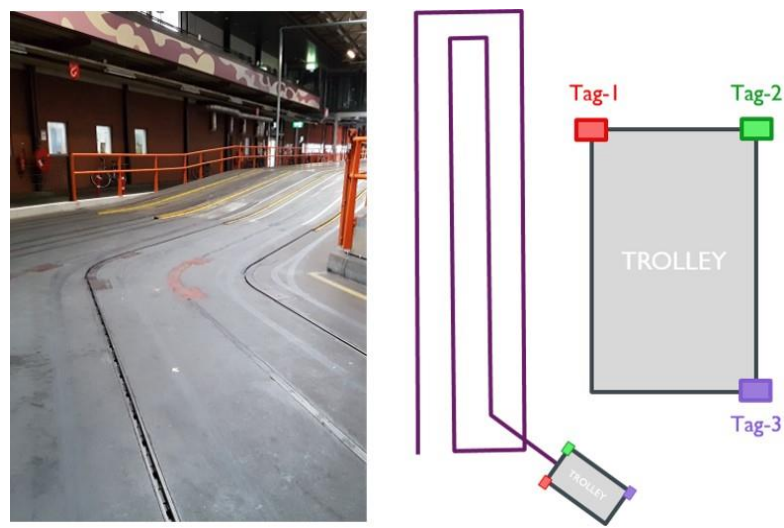

Fig. 16: Measurement Scenarios for visualization with multiple tags

The collected position data for three tags is shown in Figure 17. The darkness of the measurement points implies the elapsed time from the starting moment. By comparing the real and measured movement of the trolley, we can interpret that the system was able to approximate the track of the trolley successfully and detect the exact line the trolley was following at a certain moment. The results also show that it was able to provide a position update with approximately $80 \%$ availability performance.
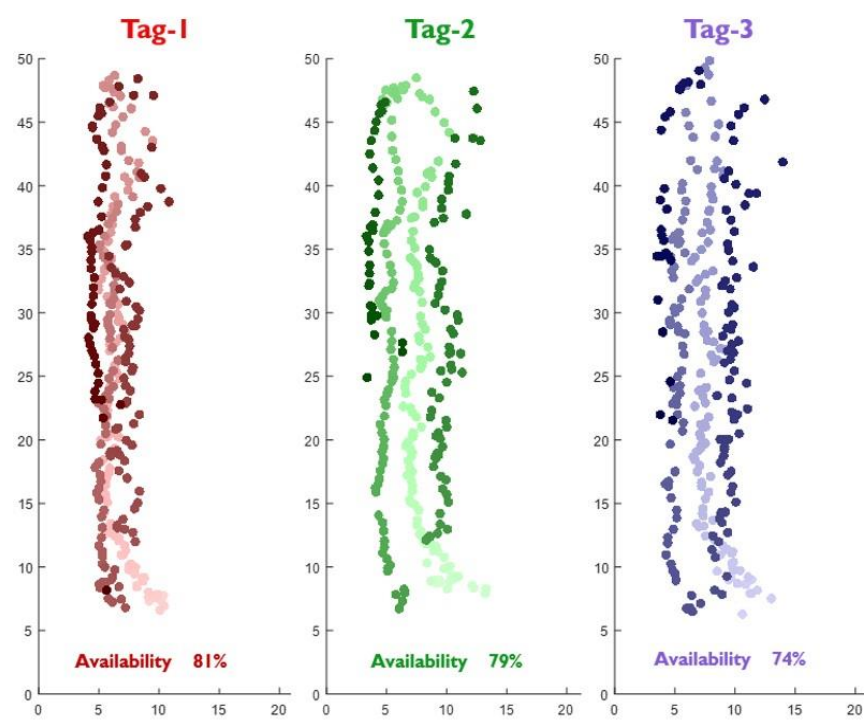

Fig. 17: Measured positions for three BLE tags moved on a Trolley

vi) Measurement Height Error (figure 11-f)

The objective of this measurement was to understand the system behavior when a tag was at a different height than the measurement height defined at system level. Normally, the system requires a measurement height setting which is fixed and used globally for every tag in the whole system. However, having a totally flat positioning area with a constant height cannot be guaranteed for some industrial sites. Therefore, it is important to know to what extend the system can tolerate the variation in tag height.

For that purpose, we set up measurement scenarios for static and mobile cases of which the results are presented in Figure 18. The measurement height was configured as $1.7 \mathrm{~m}$ from the ground, then positioning information is collected separately for tags at $1.7 \mathrm{~m}$ and $2.7 \mathrm{~m}$ height. In Figure 18 (a), green points represent the results for the correct height and the red points are the ones with the wrong height. As it can be visualized clearly, $1 \mathrm{~m}$ of tag height error resulted in a remarkable positioning error, up to $3 \mathrm{~m}$. In addition, such a drop in positioning accuracy occurs in the mobile scenario,
Figure 18 (b). Although the mobile tag was moved on a straight line, the measurement results embodied a wavy movement with varying positioning error. Further, as it gets distant from the closest anchor, the error gets larger and larger.

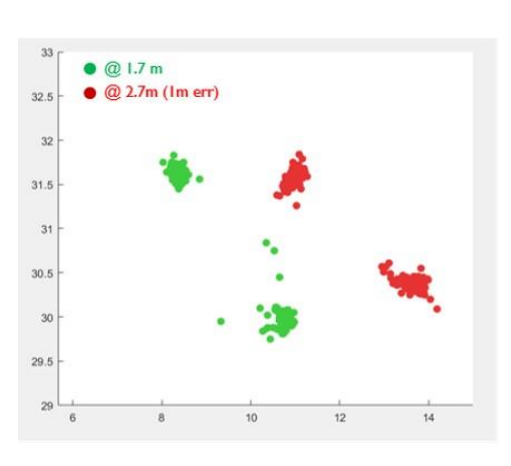

(a)

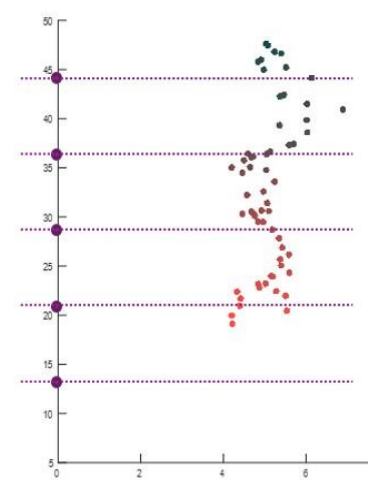

(b)
Fig. 18: Effect of Error in Measurement Height of BLE Localization System Measurement Height @1.7m, Actual Tag Height in Errornous Case @ 2.7m

(a) Fixed points, measurement height, (b) Mobile tag tracking

These results show that a variation in tag height can lead to a positioning error relatively to the distance to closest anchor and the ratio between the amount of height error and height difference between anchor and tag. Consequently, this error can be minimized by increasing the number of anchors and optimizing their position for certain areas with varying heights.

\section{OUTCOMES AND DISCUSSION}

Both UWB and BLE based localization systems are very promising and emerging technologies which can be used for very high accuracy positioning applications in industry. However, they have different strengths and weaknesses making their potential for a specific application dependent on the application's requirements.

With respect to the maturity of the systems, the BLE based localization system is the most mature one with its special antenna design, advanced localization and tracking algorithms, planning software and calibration process. On the other side, the considered UWB based technology has a very strong ranging mechanism, but lacks an advanced tracking algorithm, planning and calibration mechanisms or tools, aspects that mainly require more R\&D efforts.

In terms of accuracy, both solutions have great potential to provide very accurate (cm-level) position information thanks to their localization technique and system design. The UWB based technology delivered very accurate ranging measurements and was able to provide a sub-meter accuracy with a probability of $91 \%$. The accuracy of the system can be further increased with a more advanced positioning algorithm. For the BLE solution, its mature system design, the use of AoA technique on special antennas, accurate calibration mechanism and advanced positioning algorithm contributes to the very accurate positioning performance. However, there have to be at least two anchors at most $15 \mathrm{~m}$ separated from the tag resulting in a very dense infrastructure with a large number of anchors. For both technologies, the system requires a fixed measurement height which incurs measurement errors for deployments in sites with non-flat ground level. Also, the dynamicity of the environment has a negative effect on the accuracy of the network for both systems, however they were still able to operate even in the most dynamic areas in case of LOS. 
Concerning the scalability of the BLE solution, the use of standard BLE technology makes it compatible with standard mobile devices. By design, BLE technology defines a system capacity with a theoretical maximum of $400-500$ packets per second which means up to 400-500 location update events per second in a certain environment. The only limitation is that more tags cannot be tracked at a given time if they all happen to be active at the same time inside the same anchor detection area. However, in large deployments such as a warehouse, there can be tens of thousands of tags being tracked. On the other hand, such a large deployment requires a huge and dense infrastructure with a large number of anchors. For UWB technology, the system coverage is a lot larger than BLE. This makes it relatively easy to scale for large deployment. However, there is no advanced medium access control mechanism for multiple tags which limits the number of tags in a given area and also prevents us from evaluating its scalability in terms of the number of tags that can be supported [17]. For now, the system supports update rates between $5 \mathrm{~Hz}-10 \mathrm{~Hz}$ for a single tag. For multiple tags, the maximum update rate is divided by the number of tags.

Both the localization systems were vulnerable to the absence of LOS between the anchors and tags. For that reason, it is expected that both system can encounter a performance drop in an environment with larger machines and obstacles. In BLE based technology, the system availability decreased drastically in case a metal object prevents LOS. For UWB based technology, the system was detecting erroneous ranging due to reflected copies of the transmitted signal which lead to faulty positioning measurements in case of NLOS.

Due to the frequencies in use, both systems are robust against external interferences. The UWB solution uses 6 RFbands between $3.5 \mathrm{GHz}-6.5 \mathrm{GHz}$ which enables it to avoid any kind of interference from other RF systems such as Wi$\mathrm{Fi}$ and Bluetooth. On the other side, the BLE based solution can avoid external interferences by using proprietary channels $(2401 \mathrm{MHz}$ and $2481 \mathrm{MHz})$ at the band edges of global $2.4 \mathrm{GHz}$ ISM communication band.

In terms of system cost, the BLE system exhibits a higher installation cost due to the dense anchor network and its cabling. On the other hand, it uses small and cheap BLE chips or even standard BLE devices which decrease the amount of investment for a large number of tags. UWB based technology can provide services with a smaller number of anchors. However, both anchors and tags consist of relatively expensive and specific UWB equipment.

\section{CONCLUSION}

Despite their significant potential in industrial applications, the amount of research targeting the evaluation of indoor localization technologies in real-life industrial conditions is limited. In this work, two promising accurate indoor localization systems, UWB and BLE, were evaluated with various performance metrics for a distinct set of scenarios in a real industrial environment.

Based on the detailed performance evaluation, we can conclude that both systems have potential to be used in highly accurate positioning applications. By design, these technologies are robust against external interferences from other RF technologies. However, harsh industrial conditions have a large impact on their performance in terms of accuracy, availability and reliability. In addition, the systems were vulnerable to the absence of LOS between the anchors and tags. Therefore, these localization systems may not be sufficient enough to provide localization information in order to take critical decisions for process control applications, but they can be used for many applications including monitoring and operator navigation in industrial environments.

\section{ACKNOWLEDGMENT}

HYCOWARE is a project realized in collaboration with imec. Project partners are Egemin, Aucxis and Intation, with project support from VLAIO (Flanders Innovation and Entrepreneurship).

\section{REFERENCES}

[1] M. Holzl, R. Neumeier, and G. Ostermayer, "Localization in an industrial environment: a case study on the difficulties for positioning in a harsh environment", International Journal of Distributed Sensor Networks, vol. 2015, 2015.

[2] L. Mainetti, L. Patrono and I. Sergi, "A Survey on Indoor Positioning Systems", SoftCOM 2014: 111-120.

[3] Z. Farid, R. Nordin and M. Ismail, Recent Advances in Wireless Indoor Localization Techniques and System, Journal of Computer Networks and Communications, vol. 2013, 2013.

[4] E. Tanghe, W. Joseph, L. Verloock, L. Martens, Henk Capoen, Kobe Van Herwegen, and Wim Vantomme, "The Industrial Indoor Channel: Large-Scale and Temporal Fading at 900,2400, and $5200 \mathrm{MHz}$ ", IEEE Trans. Wireless Commun., vol. 7, no. 7, pp. 2740-2751, July 2008.

[5] X. Gong, J. Trogh, Q. Braet, E. Tanghe, P. Singh, D. Plets, D. Deschrijver, J. Hoebeke, T. Dhaene, L. Martens, and W. Joseph, "A Measurement-Based Wireless Network Planning, Monitoring, and Reconfiguration Solution for Robust Radio Communications in Indoor Factories", IET Science, Measurement \& Technology, 2015

[6] A. Alarifi, A. Al-Salman, M. Alsaleh, A. Alnafessah, S. Al-Hadhrami, M. Al-Ammar, and H. Al-Khalifa, "Ultra Wideband Indoor Positioning Technologies: Analysis and Recent Advances," Sensors, vol. 16, no. 5, pp. 707, 2016 .

[7] S. Woo, S. Jeong, E. Mok, L. Xia, C. Choi, M. Pyeon, and J. Heo, "Application of WiFi-based indoor positioning system for labor tracking at construction sites: A case study in Guangzhou MTR," Automation in Construction, vol. 20, pp. 3-13, Jan 2011.

[8] E. Dahlgren, H. Mahmood, "Evaluation of indoor positioning based on Bluetooth Smart technology", June 2014.

[9] T. Van Haute, E. De Poorter, P. Crombez, et al. "Performance analysis of multiple indoor positioning systems in a healthcare environment", International Journal of Health Geographics. 2016.

[10] R. Faragher and R. Harle, "An Analysis of the Accuracy of Bluetooth Low Energy for Indoor Positioning Applications", 27th International Technical Meeting of The Satellite Division of the Institute of Navigation, September 2014, pp. 201-210

[11] T. Van Haute, E. De Poorter, I. Moerman, F. Lemic, V. Handziski, A. Wolisz, N. Wirstrom and T. Voigt, "Comparability of RF-based Indoor Localization Solutions in Heterogeneous Environments: An Experimental Study", 2015.

[12] D. Dardari, A. Conti, U. J. Ferner, A. Giorgetti, and M. Z. Win, "Ranging with ultrawide bandwidth signals in multipath environments," Proc. IEEE, vol. 97, no. 2, pp. 404-426, Feb. 2009.

[13] L. Zwirello, M. Janson, C. Ascher, U. Schwesinger, G. F. Trommer and T. Zwick, "Localization in industrial halls via ultra-wideband signals" 7th Workshop on Positioning, Navigation and Communication (WPNC '10), March 2010.

[14] T. Van Haute, B. Verbeke, E. De Poorter and I. Moerman, "Optimizing Time of Arrival Localization Solutions for Challenging Industrial Environments", IEEE Transactions on Industrial Informatics, (Volume PP, Issue 99), 2016.

[15] Royal Flora Holland company website, Retrieved on January 10, 2017, from https://www.royalfloraholland.com.

[16] M. S. Grewal, A. P. Andrews, "Kalman Filtering: Theory and Practice with MATLAB", Wiley-IEEE Press, 2014

[17] M. Ridolfi, S. Van de Velde, H. Steendam, E. De Poorter, "WiFi adhoc mesh network and MAC protocol solution for UWB indoor localization systems", Symposium on Communications and Vehicular Technologies (SCVT), 2016 